bilder erzeugt, deren Umrisse mit der ebenfalls projizierten Drehachse des Kristalls aufgezeichnet werden.

Die Abstände der Umrißlinien von der Drehachse und die Drehwinkel $\varphi$ erlauben in äquidistanten Ebenen senkrecht zur Drehachse die Berechnung von Polygonen, die den Kristall umhüllen. Sie stellen den Kristallumriß um so genauer dar, je mehr Schattenrisse zu ihrer Konstruktion beigetragen haben. Aus den Polygonen wird ein dichtes Netz von Punkten in einem körperfesten $x y z$-Koordinatensystem berechnet. Diese Beschreibung der Oberfläche ist besonders geeignet, um nach dem numerischen Integrations-Verfahren von HaMIITON [1] die Absorption und Extinktion zu ermitteln. Dazu werden die Punkte in ein Koordinatensystem transformiert, dessen Achsen parallel zum ein- und ausfallenden Röntgenstrahl und der zu diesen beiden Strahlen senkrechten Richtung sind. Um den Einfluß der Polarisation auf die Extinktion zu berücksichtigen, wird der einfallende Strahl in einen parallel und einen senkrecht zur Beugungsebene polarisierten aufgespalten und für beide getrennt die Integration durchgeführt.

Ein Fortran-Programm, das zuerst das Oberflächennetz und daraus Absorption- und Extinktionsfaktoren für WeißenbergReflexintensitäten berechnet, befindet sich im Teststadium.

Der Deutschen Forschungsgemeinschaft dankt Herr Dieriks für ein Stipendium.

Eingegangen am 6. Februar 1967

[1] Hamilton, W. C.: Acta Cryst. 10, 629 (1957); 16, 609 (1963).

\section{Enzymatische Untersuchungen an glomerulären Basalmembranen}

G. H. THOENES

Max-Planck-Institut für Immunbiologie, Freiburg i. Br.

Glomeruläre Basalmembranen (BM) der Niere enthalten größere Mengen Hydroxyprolin und Hexosamin [1-3]. Es ist daher anzunehmen, daB BM sich aus collagenartigetn Material und Polysacchariden zusammensetzen. Spezifische Antikörper gegen das nephrotoxische Antigen der BM (AntiBM) fixieren nach Injektion in den homologen Nierenspender an der BM. Die nachfolgenden Untersuchungen befassen sich mit der Identifizierung des nephrotoxischen Antigens mit Hilfe enzymatischer Methoden.

Tabelle. Nierenfixierende Antikörperquoten gegen intakte und enzymbehandelte Basalmembranen (bestimmt als Protein-J ${ }^{131}$-Aktivität, nievenfixiert. - Doppelbestimmungen)

\begin{tabular}{lll}
\hline Antiserum gegen & $\begin{array}{l}\% \text { Gesamt-IgG, } \\
\text { nierenfixiert }\end{array}$ & $\begin{array}{l}\% \text { Nierenspezifisches } \\
\text { IgG, fixiert }\end{array}$ \\
\hline Intakte BM (Norm) & $2,22 / 2,24$ & $2,03 / 2,03$ \\
Collase-BM & $1,19 / 1,54$ & $1,00 / 1,33$ \\
Hyase-BM & $1,09 / 1,22$ & $0,90 / 1,01$ \\
Kontrolle: Normalserum & $0,19 / 0,21$ & - \\
\hline
\end{tabular}

Unter Einhaltung identischer Versuchsbedingungen (Dosis, Immunisierungsschema, Körpergewicht; etc.) wurden Kaninchen gegen folgende Ratten-BM immunisiert: 1. Intakte BM; 2. Collagenase-behandelte BM (Collase-BM); 3. Hyaluronidase-behandelte BM (Hyase-BM). Die Enzympräparate $[4]$ waren auf Substratspezifität geprüft. Nach Immunisierung wurden gleiche Teile individueller Antisera, getrennt nach Versuchsgruppen, gepoolt und auf nephrotoxische Eigenschaften untersucht. Die aus vorgenannten Serumpools gewonnenen IgG-Fraktionen * wurden mit J ${ }^{131}$ markiert [5]. 3-4 Ratten pro Gruppe erhielten i. v. Injektionen von IgG (ca. $500 \mu \mathrm{g})$ mit spezifischen Antikörperquoten gegen intakte [6] oder die enzymatisch behandelten BM. Als Kontrolle dienten Ratten, injiziert mit normalem IgG. Nach 3 Tagen wurden die Tiere getötet, die Nieren blutfrei gespült, und im $\gamma$-Scintillationszähler die in beiden Nieren jeweils gebundene Aktivität bestimmt. Die Meßwerte der 3-4 Tiere jeder Gruppe wurden gemittelt und sind in der Tabelle wiedergegeben.

Durch enzymatischen Abbau konnte die Antigenität der BM im Vergleich zur intakten BM so vermindert werden, daß nur noch eine Antikörper-Ausbeute von ca. 50\% der Norm erzielbar war (Immunisierungsdauer 6 Monate!). Hyase verändert die nephrotoxische Qualität des Antigens stärker als Collase. Da einerseits das Gewicht der zum Abbau eingesetzten BM von Hyase kaum, von Collase jedoch stärker reduziert wurde, andererseits ohne Rücksicht auf den Gewichtsverlust die Totalrückstände aus eingesetzten gleichen Mengen BM zur Immunisierung benutzt wurden, wurden effektiv mehr Hyase-BM injiziert als Collase-BM. Trotzdem enthielten die Antisera gegen Hyase-BM weniger spezifische Antikörper als die durch Collase-BM erzeugten.

Über die, auch teilweise unspezifische, Wirkung der Collase hinaus führt die sicher spezifische Hyase $z u$ einem Abbau antigener Substanzen, die für die Erzeugung nephrotoxischer Antikörper und deren biologische Aktivität von Bedeutung sind. Aus den Befunden wird geschlossen, daß das nephrotoxische Antigen im wesentlichen vom Polysaccharid verkörpert wird. Versuche zur Darstellung des reinen Antigens sind im Gang und scheinen die hier dargestellten Befunde zu bestätigen [ 7 u. a.].

Die Untersuchungen und ihre Fortführungen, die von der Deutschen Forschungsgemeinschaft ermöglicht wurden, werden ausführlich veröffentlicht werden.

Eingegangen am 16. Januar 1967

* $\mathrm{IgG}=\mathrm{yG}=$ hochgereinigtes Gammaglobulin.

[1] KeFAIIDES, N. A., 11. R. J. WINZLER: Biochem. 5, 702 (1966), [2] Dische, R. M., et al.: Biochem. Biophys. Res. Commun. 20,63 (1965). - [3] Misra, R. P., u. L. B. Berman: Proc. Soc. exp. Biol. Med. 122, 705 (1966). - [4] Worthington Biochemical Corp./ USA. - $[5]$ McConAhey, P. J., v. F. J. Dixon: Intern. Arch. Allergy Appl. Immunol. 29, 185 (1966). - [6] UNanUe, E. R., u. F. J. Dixon: J. exp. Med. 121, 697 (1965). [7] Smrbata, S., et al.: Jap. J. exp. Med. 36, 143 (1966).

\section{Selektive Erzeugung von Darmkrebs bei Ratten durch 1,2-Dimethyl-hydrazin}

H. Druckrey, R. Preussmani, F. Matzkies und

S. IVANKOVIC

Forschergruppe Praeventivmedizin am Max-Planck-Institut für Immunbiologie in Freiburg

1,2-Diaethyl-hydrazin [1] erzeugt ebenso wie Azo- und Azoxy-äthan [2] nach wiederholter subcutaner Injektion an Ratten speziell neuroektodermale Tumoren in der Nasenhöhle

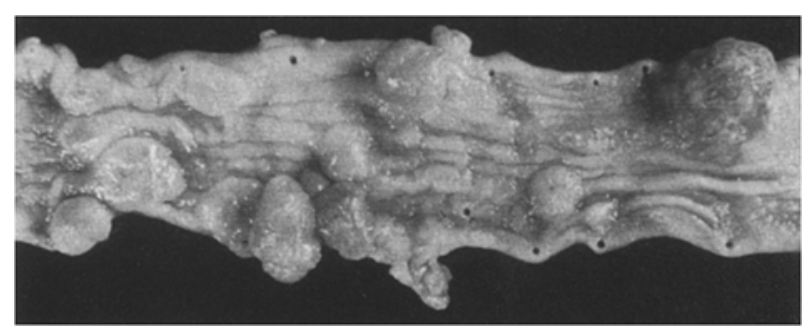

Fig. 1. Multiple Adenocarcinome im Colon einer Ratte, erzeugt durch s. c. Injektion von 1,2-Dimethyl-hydrazin, $21 \mathrm{mg}$ pro $\mathrm{kg}$ einmal i. d. Woche. Induktionszeit 231 Tage

bzw. im Gehirn, Brustkrebs und Leukämien. Die Substanzen haben keine, "dirckte" carcinogene Wirkung, sondern bedürfen einer metabolischen Aktivierung, wahrscheinlich durch oxydative Desalkylierung zum Diazoalkan. Danach waren auch in dieser Stoftklasse organotrope Wirkungen zu erwarten, wie wir sie bei Dialkyl-nitrosaminen nachweisen konnten [3]. Hier sei über die selektive Erzeugung von Colon- und Rectum-Carcinomen berichtet. Sie wurde bereits von LAgUEUR nach Gabe von Cycasin und seinem Aglycon Methylazoxymethanol an Ratten beobachtet [4].

1,2-Dimethyl-hydrazin (DNH) wurde durch Methylierung von 1,2-Dibenzoyl-hydrazin und Abspaltung der Acyl-Reste als Dihydrochlorid ( $\mathrm{Fp} 165-167^{\circ} \mathrm{C}$ ) hergestellt. Für die Versuche benutzten wir auf pH 6,5 neutralisierte wäßrige Lösungen. Alle Dosierungen beziehen sich auf die Base. Die Bestimmung der mittleren letalen Dosis (LD 50) ergab subcutan 215 und oral $100 \mathrm{mg}$ pro kg. 